\title{
Do Herbig Ae Stars Pulsate?
}

\author{
M. Marconi ${ }^{1}$, V. Ripepi ${ }^{1}$, J. Alcalá ${ }^{1}$, E. Covino ${ }^{1}$, L. Terranegra ${ }^{1}$, \\ F. Palla ${ }^{2}$
}

\begin{abstract}
We present some preliminary results of a Strömgren photometric survey searching for radially pulsating variables among Herbig Ae stars. These young objects are expected to show a characteristic $\delta$ Scuti variability when entering the instability strip along their pre-main sequence evolutionary tracks, according to the theoretical predictions by Marconi \& Palla (1998). We identified at least five pulsating Herbig Ae stars with periods in the expected range. On the basis of these encouraging first results, we foresee both new more detailed observations and accurate comparisons with theoretical models.
\end{abstract}

\section{Introduction}

Herbig Ae intermediate mass stars are expected to pulsate since they cross the instability strip of more common post-main sequence $\delta$ Scuti stars during their pre-main sequence (PMS) evolution. The identification of a few PMS objects pulsating as $\delta$ Scuti stars (Breger 1972; Kurtz \& Marang 1995) has supported this idea. From the theoretical point of view, $\delta$ Scuti nonlinear pulsation models along evolutionary PMS tracks have been computed by Marconi \& Palla (1998), and the theoretical PMS instability strip for the first three radial modes of pulsation has been produced. These results stimulated a photometric investigation of a sample of Herbig Ae stars to search for a $\delta$ Scuti variability.

\section{The Photometric Survey}

The main goals of our photometric investigation of Herbig Ae stars were to identify new PMS $\delta$ Scuti and to constrain theoretical predictions, improving our knowledge of the structural properties of Herbig stars. The selected sample consisted of 8 Herbig Ae stars in the range A5 to F5; observations were made with the $0.9-\mathrm{m}$ Dutch and $1.5-\mathrm{m}$ Danish telescopes at La Silla during periods \#62 and \#63. Strömgren (uvby) photometry was performed in order to derive information about effective temperature and surface gravity through the adoption of model atmospheres. Data reduction was carried on by using the MIDAS package.

\footnotetext{
${ }^{1}$ Osservatorio Astronomico di Capodimonte, Via Moiariello, 16, I-80131 Napoli, Italy

${ }^{2}$ Osservatorio Astrofisico di Arcetri, Largo E. Fermi, 5, I-50125 Firenze, Italy
} 

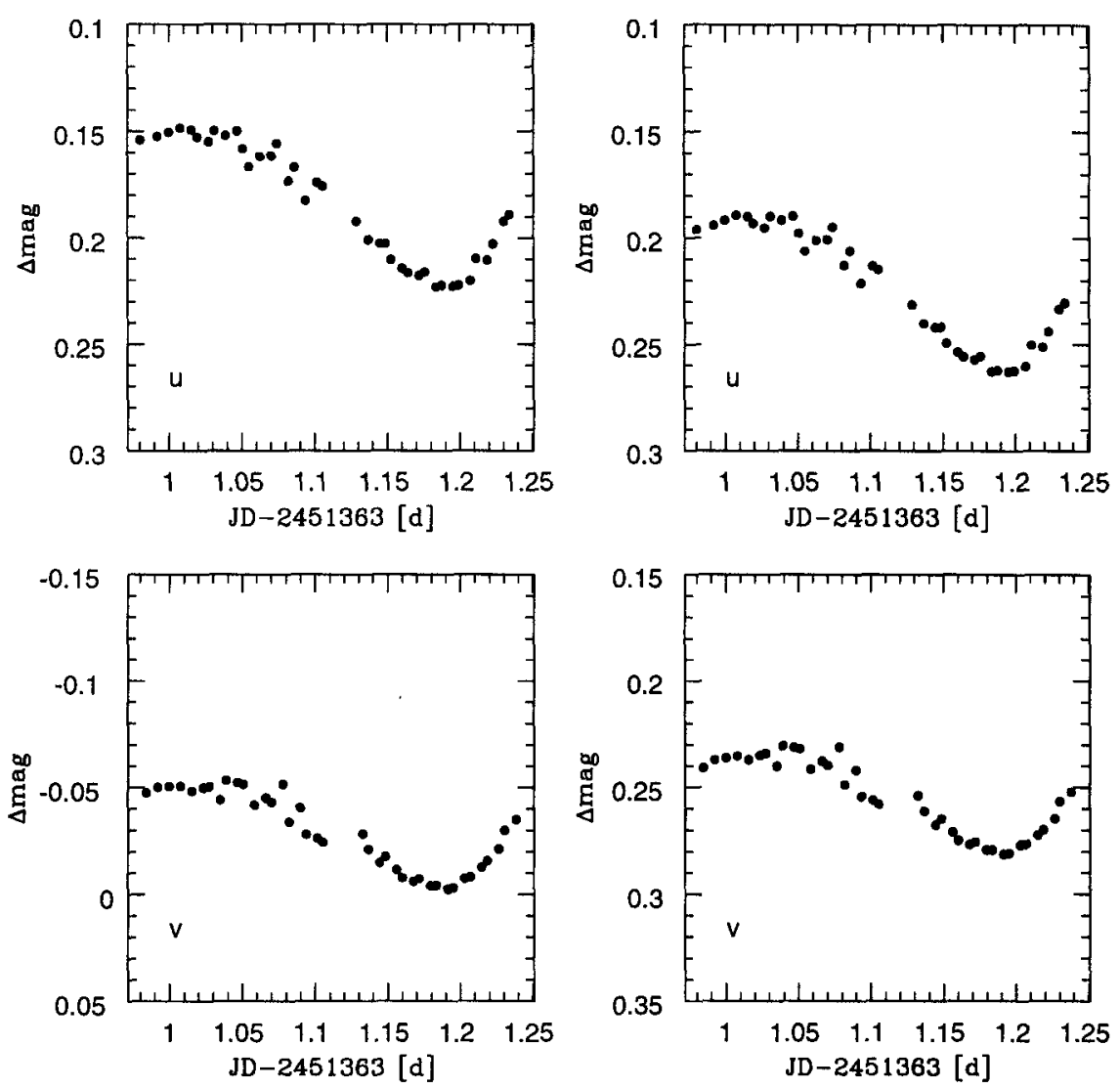

Figure 1. Temporal variation of the magnitude difference $\Delta \mathrm{mag}$ between $\mathrm{AK}$ Sco and the comparison star in the Strömgren filters $u$ and $v$. Left and right panels refer to two different comparison stars.

\section{Results}

Five candidates have been found to show photometric variability on the expected time scale: V351 Ori, V346 Ori, HD 35929, BN Ori and AK Sco. Preliminary results are presented for AK Sco (see Fig. 1).

\section{References}

Breger, M. 1972, ApJ, 171, 539

Kurtz, D. W. \& Marang, F. 1995, MNRAS, 276, 191

Marconi, M. \& Palla, F. 1998, ApJ, 507, L141 